\title{
Methodology of Wavelets in Relativistic Heavy Ion Collisions in One Dimensional Phase Space ( $\eta$-Space)
}

\author{
Vyacheslav V. Lyashenko', M. Ayaz Ahmad², Zhanna V. Deineko³ ${ }^{3}$ Mir Hashim Rasool ${ }^{4}$ \\ ${ }^{1}$ Department of Informatics, Kharkiv National University of Radio Electronics, Kharkiv, Ukraine \\ ${ }^{2}$ Physics Department, Faculty of Science, University of Tabuk, Tabuk, Saudi Arabia \\ ${ }^{3}$ Department of Media Systems and Technology, Kharkiv National University of Radio Electronics, Kharkiv, Ukraine \\ ${ }^{4}$ Department of Physics, Islamic University of Science and Technology, Awantipora, India \\ Email: lyashenko.vyacheslav@mail.ru,mayaz.alig@gmail.com,dgv_zhanna@ukr.net, hrasool23@gmail.com
}

How to cite this paper: Lyashenko, V.V., Ahmad, M.A., Deineko, Z.V. and Rasool, M.H. (2017) Methodology of Wavelets in Relativistic Heavy Ion Collisions in One Dimensional Phase Space ( $\eta$-Space). Journal of High Energy Physics, Gravitation and Cosmology, 3, 254-266. https://doi.org/10.4236/jhepgc.2017.32021

Received: February 9, 2017

Accepted: March 24, 2017

Published: March 27, 2017

Copyright $\odot 2017$ by authors and Scientific Research Publishing Inc. This work is licensed under the Creative Commons Attribution International License (CC BY 4.0).

http://creativecommons.org/licenses/by/4.0/

\begin{abstract}
An attempt has been made to apply the wavelet methodology for the study of the results of the chaotic behavior of multiparticle production in relativistic heavy ion collisions. We reviewed the data that describes the collisions of relativistic heavy ion for the case $\eta$-space in $1-D$ phase space of variable. We compared the experimental data and UrQMD data using wavelet coherency. We discussed the results of the comparison.
\end{abstract}

\section{Keywords}

Wavelet Coherence, Morlet Wavelet, Heavy Ion Collisions, Reaction Dynamics, Phase Space, Normalized Factorial Moments

\section{Introduction}

Particle physics is the science of the fundamental structure of matter. One of the directions of particle physics is the study of high-energy nuclear matter. The interest in the study of high-energy nuclear matter has increased many folds due to the possibility of studying unstable states of nuclear matter under extreme condition of high energy density and high temperature. The study of non-statistical fluctuations in relativistic nuclear collisions has recently also attracted a great deal of attention due to the possibility of extracting important information about the mechanism of multiparticle production in such collisions [1] [2]. The multiplicity of charged particles in high energy nucleus-nucleus interactions is an important parameter which indicates how many particles are produced in that interaction. In the same time the multiplicity distributions of produced particles 
help in learning the interaction mechanism [3] [4].

But it is appropriate to study the field in terms of theoretical and the experimental points of view. This will help understand the relationship between theory and experiment, understand the complicated moments which may arise. Theory predicts phenomenon, which can be verified by experiments, and experiments very often provide new insight through unexpected results, which in turn lead to the improvement in theoretical description. In the present paper, an attempt has been made to find the relationship between the stability of a theoretical model and experiment to study the collisions of relativistic heavy ion. This exercise has been made to perform the study of $(E$-by- $E)$ spatial fluctuations of relativistic shower particles produced in the collisions of ${ }^{28} \mathrm{Si}+E m$ at energy $14.6 \mathrm{~A} \mathrm{GeV}$ in 1- $D$ phase space of $X$-variable (the case $\eta$-space) [5]. The findings are compared with the predictions of Ultra-relativistic Quantum Molecular Dynamics (UrQMD) model [6] [7].

To solve this problem we use wavelets ideology [8] [9]. This is due to the fact that various processes and the phenomenon can have quite difficult structure, and contain local features of the various form and time extent. Wavelet-analysis transforms the original data to hierarchical structure by means of the wavelet transformations which results to the set of wavelet coefficients. If the signal is discontinuous, only those wavelets will have high amplitudes, where the maximum value will appear near the discontinuity point, which will allow detecting image contour. At the same time, discontinuity point is a sharp intermittent transition during some process. Quantitatively, it can be estimated by the value of the first derivative of such process, taking into consideration that the first derivative of intermittent transitions is very high. If the transition is in the form of discontinuity point, then the first derivative tends to infinity. The sharper the transition, the higher the derivative value is. Smooth transitions will have small derivative values. This allows us to determine the presence of special characteristics of the analyzed one, as well as the point where these characteristics may arise [10] [11]. Thus methodology of wavelet analysis allows taking into consideration the particular characteristics study by decomposing source data into a plurality of approximate and detailed coefficients. This allows conducting a detailed analysis of the original data.

\section{Some Experimental Details}

To obtain the data, FUJI nuclear emulsion pellicles were irradiated horizontally with a beam of $28 \mathrm{Si}$ nuclei at $14.6 \mathrm{~A} \mathrm{GeV}$ at Alternating Gradient Synchrophasotron (AGS) of Brookhaven National Laboratory (BNL), New York, USA have been used. The nuclear emulsion experiment is a versatile detector for the study of nuclear reactions in high energy heavy ion collisions. It has the ability to detect and identify the secondary charged particles in the outlet channel of nuclear reactions. The method of line scanning has been adopted to scan the stacks, which was carried out carefully using Japan made NIKON (LABOPHOT and Tc-BIOPHOT) high-resolution microscopes with $8 \mathrm{~cm}$ movable stage using $40 \times$ 
objectives and 10x eyepieces by two independent observers, so that the bias in the detection, counting and measurements can be minimized. The interactions due to beam tracks making an angle $<2^{\circ}$ to the mean direction and lying in emulsion at depths $>35 \mu \mathrm{m}$ from either surface of the pellicles were included in the final statistics [5] [12].

\section{Mathematical Formalism for Obtaining Data}

In order to perform a meaningful analysis of chaoticity, normalized cumulative variable $(X(\eta))$ were used to reduce the effect of non-uniformity in single charged particle distributions. At the same time, we consider only one component in 1- $D$ phase space of $X$-variable (this is the case $\eta$-space). The single charged particle density distribution is not flat in the analysis of the fluctuation in phase space variable. This non-uniformity of the particle spectra influences the scaling behaviour of scaled factorial moments. Bialas and Gazdzicki [13] proposed a method to construct a set of variables, which drastically reduces the distortion of intermittency due to the non-uniformity of single particle density distribution. According to them, the new scaled variable $X(\eta)$ is related to the single particle density distribution $\rho(\eta)$ by the following relation:

$$
X(\eta)=\frac{\int_{\eta_{1}}^{\eta} \rho\left(\eta^{\prime}\right) \mathrm{d}\left(\eta^{\prime}\right)}{\int_{\eta_{1}}^{\eta_{2}} \rho\left(\eta^{\prime}\right) \mathrm{d}\left(\eta^{\prime}\right)},
$$

where, $\rho(\eta)=(1 / N) \mathrm{d} n / \mathrm{d} \eta$ is the single particle pseudo rapidity density distribution of the shower particles and $\eta_{1}$ and $\eta_{2}$ are the two extreme points in the distribution $\rho(\eta)$ (or $\eta_{1}=\eta_{\min }, \eta_{2}=\eta_{\max }$ ). Should also be noted that, in terms of new scaled variable $(X(\eta))$ the single particle density distribution is always uniform in between $X=0$ and 1 .

Various experimental efforts have established the existence of the empirical phenomenon of "intermittency" in multiparticle production using normalized scaled factorial moments. On the basis of bin averaging the normalized scaled factorial moments of the order of $\mathrm{q}$ is defined in vertical form as [5] [12] [13]:

$$
F_{q}^{V}(\delta \eta)=\frac{1}{M^{d}} \sum_{m=1}^{M^{d}} \frac{\left\langle n_{m}^{q}\right\rangle}{\left\langle n_{m}\right\rangle^{q}}
$$

and its horizontal form is defined as [5] [12] [13]:

$$
F_{q}^{H}(\delta \eta)=\left\langle\frac{1 / M^{d} \sum_{m=1}^{M^{d}} n_{m}^{q}}{\left(\langle n\rangle / M^{d}\right)^{q}}\right\rangle,
$$

where, $n_{m}^{q}=n_{m}\left(n_{m}-1\right) \cdots\left(n_{m}-q+1\right)$, and also bracket $\langle\cdots\rangle$ of Equation (3) indicates the average over all events in the whole data sample; $n_{m}$ is the number of relativistic charged particles in the $m^{\text {th }}$ bin, m can take values from 1 to $M$ and $N$ represents the total multiplicity of charged shower particles in a particular event in 
the pseudo-rapidity interval $\Delta \eta=M \delta \eta \quad\left(\right.$ or $\delta \eta=\left\{X\left(\eta_{\max }\right)-X\left(\eta_{\min }\right)\right\} / M$ ).

Recently, Cao and Hwa first introduced to measure the spatial pattern of particles in an event using normalized factorial moments associated with it. In contrast to the horizontally averaged vertical moments, $F_{q}^{V}$ and vertically averaged horizontal moments, $F_{q}^{H}$ of the $q^{\text {th }}$ order, they define event factorial moments as [14]:

$$
F_{q}^{(e)}=\left[\frac{1}{M} \sum_{m=1}^{M} n_{m}\left(n_{m}-1\right) \cdots\left(n_{m}-q+1\right)\right] \times\left(\frac{1}{M} \sum_{m=1}^{M} n_{m}\right)^{-q},
$$

where, $M$ is the partition number in phase space, $n_{m}$ is the number of shower tracks producing particles falling into the $m^{\text {th }}$ bin and $q=2,3,4 \cdots$ is the order of the moment.

The event factorial moments, $F_{q}^{e}$, fluctuates from event-to-event, and the degree of fluctuation can be estimated from the probability distribution $P\left(F_{q}^{e}\right)$ over all events. One can obtain a distribution $P\left(F_{q}^{e}\right)$ for the whole sample of events. In the given situation, a normalized factorial moment of a single event is defined as [5] [12]:

$$
\begin{gathered}
\eta_{q}(M)=\frac{F_{q}^{e}(M)}{\left\langle F_{q}^{e}(M)\right\rangle}, \\
\left\langle F_{q}^{e}(M)\right\rangle=\frac{1}{N_{e v}} \sum_{e=1}^{N_{e v}} F_{q}^{e}(M),
\end{gathered}
$$

where, $N_{e v}$ is the number of events in a sample and $F_{q}^{e}(M)$ represents the event factorial moment describing the spatial pattern of an event. It is important to mention that the SFMs introduced to study the intermittency or fractality in multiparticle production is only an estimate of the mean of the probability distribution of event by event Scaled factorial moment (SFM), $P\left(F_{q}^{e}\right)$. It should be realized that the simple mean procedure, apart from its clear advantages, suppresses a lot of important information about the fluctuations of spatial patterns of final state of multiparticle production. In particular, some interesting effects present only in a part of sample of events produced in high-energy collisions, may be lost.

In order to quantify the degree of the fluctuations, a new normalized moment related to the chaotic nature of the system is defined as [14] [15] [16]:

$$
C_{p, q}(M)=\left\langle\eta_{q}^{p}(M)\right\rangle=\frac{1}{N_{e v}} \sum_{e=1}^{N_{e v}} \eta_{q}^{p}(M),
$$

where, $p$ is any positive real number, it should not be negative, $F_{q}^{e}(M)$ may vanish for some events, if $p$ is negative.

The other relevant details may be seen in works [5] [12].

Thus, we examine the relationship between, $C_{p, q}(M)$ and $M$ (this technique of erraticity moments [5]) for order of moments $q=2-4$, and for $p=$ $0.5,0.9,1.2,1.4$ and 1.6 for the present experimental data of nucleus-nucleus collisions and with the predictions of Ultra-relativistic Quantum Molecular Dynamics (UrQMD) model. The findings in the forms of the pictorial graphs have 
been plotted between the natural $\log$ of normalized erraticity moments $\ln C_{p, q}$ as a function of $\ln M$ for $\eta$-phase spaces respectively at energy $14.6 \mathrm{~A} \mathrm{GeV}$. These data can be found in [5] (see Figure 1).

We can see (see Figure 1), which is experimental data and UrQMD data can be varied in a certain range. Thus, we have a few of time series. These time series should be compared using the ideology of wavelets. For this we will use the wavelet coherency.

\section{Methods of Wavelet Analysis as Tool to Study Time Series}

The basis of formal generalization of continuous wavelet transformation on the time interval $t$ is converting the input time series $x(t) \in L^{2}(R)$ using wavelet parent $\varphi(t)$ [17] [18] [19]:

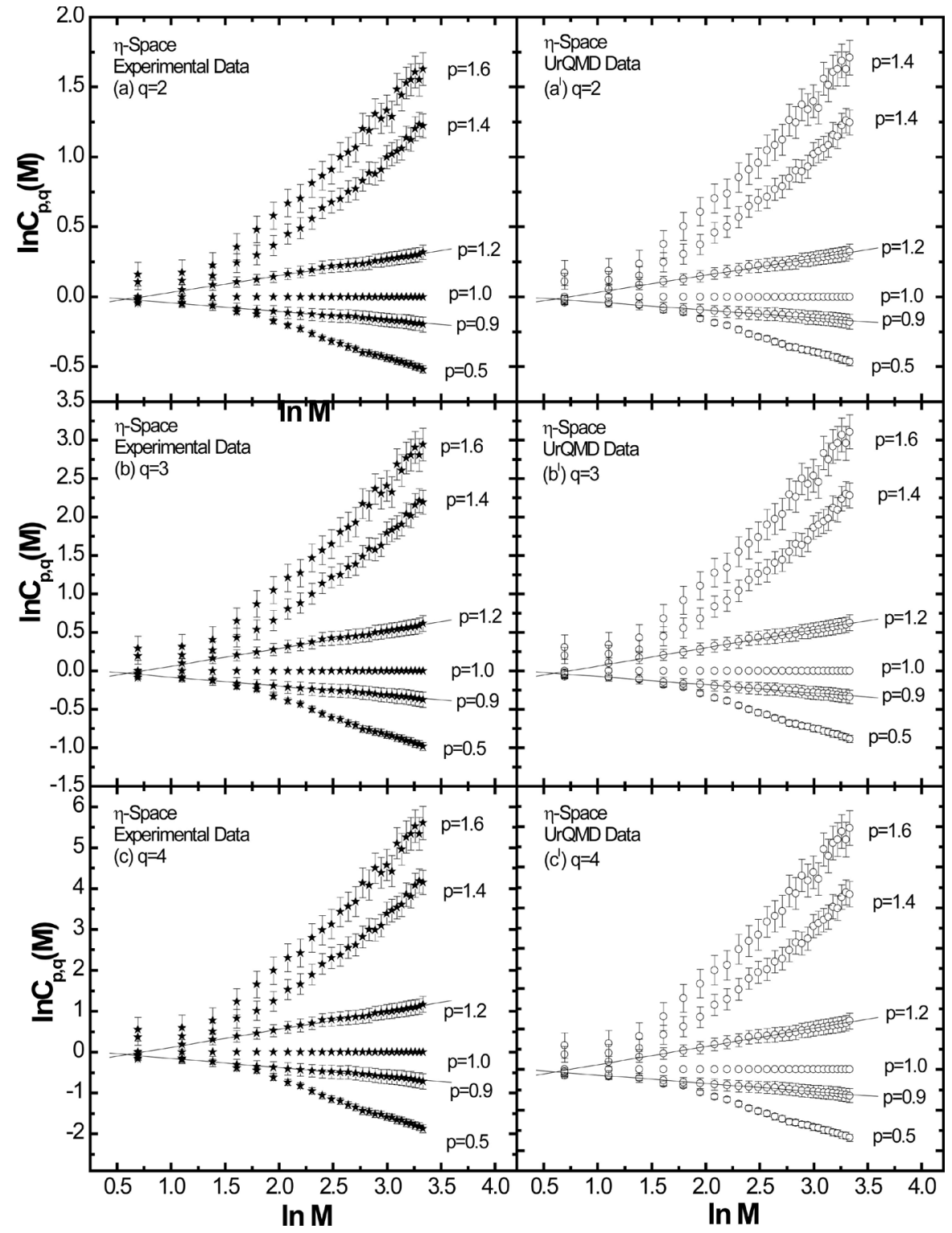

Figure 1. Variations of $\ln C_{p, q}(M)$ as function of $\ln M$ in $\eta$-space (1D) in the collisions of ${ }^{28} \mathrm{Si}+E m$ at energy $14.6 \mathrm{~A} \mathrm{GeV}$. 


$$
W_{x}(u, s)=\int_{-\infty}^{+\infty} x(t) \frac{1}{\sqrt{s}} \varphi\left(\frac{t-u}{s}\right),
$$

where $\frac{1}{\sqrt{s}}$ denotes a normalization, $u$ is a location parameter, $s$ is a scale parameter,

$$
\int_{-\infty}^{\infty} \varphi(t) \mathrm{d} t=0 .
$$

In this case, feasibility of wavelet analysis in time series study is determined by the fact that the method of wavelet analysis allows to discover the local features of the studied time series due to the decomposition of the input data [18] [19]. That is, the wavelet transformation defines the hierarchical structure of the input of the studied time series. Underlying this statement is the fact that information flows, generated by fractal time series have properties detected by wavelet transform, making it even more informative [10]. Then wavelet transform allows adding input characteristics of time series that are investigated.

In addition, the empowerment of study time series using wavelet analysis methodology promotes the use of various procedures of wavelet transformation: even-scaled analysis, cross wavelet transformation, wavelet coherence. Then wavelet analysis methodology has been widely used in the disclosure of dynamics of time series that define various data [10] [11].

One of the main wavelet transformation methods used for generalized cross-reference analysis between different time series is wavelet coherence. It allows calculating local correlation of two time series in a region of time-frequency. It uses the following formalized model: wavelet coherence as the squared absolute value of the smoothed cross wavelet spectra $W_{x y}(u, s)$ (time $(x)$ and frequency $(y)$ of the time series), normalized by the product of the smoothed individual wavelet power spectra of each series [20] [21]:

$$
R^{2}(u, s)=\frac{\left|Q\left(s^{-1} W_{x y}(u, s)\right)\right|}{Q\left(s^{-1}\left|W_{x}(u, s)\right|^{2}\right) Q\left(s^{-1}\left|W_{y}(u, s)\right|^{2}\right)},
$$

where $Q$ is a smoothing operator.

We use Morlet wavelet that is a complex wavelet with a good time-frequency localization, as a parent one [20]-[26]. The squared wavelet coherency coefficient is in the range $0 \leq R^{2}(u, s) \leq 1$, values close to zero indicate weak correlation, while values close to one are evidences of strong correlation. Thus, wavelet coherency analysis enables interconnection between the studied time series and analyzes the frequency of such communications.

\section{Result and Discussion}

We consider the wavelet coherency for each pair of time series of the natural log $C_{p, q}(M)$ (experimental data and UrQMD data) for order of moments $q=2-4$, and for $p=0.5,0.9,1.2,1.4$ and 1.6.

In Figures 2-6 you can check the results of wavelet coherence between se- 
lected time series. Each of the following figures indicated a sepa-rate group of time series that match each other. This comparison is displayed in the time-frequency plane. In this case the time scale is equal to the consistent change of values $\operatorname{Ln}(M)$ (these changes represented a sequence number $1,2, \cdots)$. The correspondence between a sequence number and value of $\operatorname{Ln}(M)$ is shown in Table 1.

On the vertical axis are the weighted characteristics of the analyzed data series in frequency space. Along each of the figures importance scale is presented as separate columns for reflections. Maximum reflection is speaks about full coherence between the experimental data and UrQMD data. Defined lines are a manifestation of localization for individual irregularities within studied time series according to importance of irregularities. In general, each point of wavelet reflects shown in Figures 2-6 is their value in the time-frequency space, which is calculated through wavelet transformation.

The phase difference, indicated by arrows, gives us details about delays of oscillation of the two examined time series. Arrows pointing to the right (left) when the time series are in-phase (anti-phase) or are positively (negatively) correlated. Arrow pointing up means that the first time series leads the second one, arrow pointing down indicates that the second time series leads the first one. In our case, these data suggest discrepancy of the experimental data according to the model.

We see that with increasing values $q$ of unbalance occurs between the experimental data and UrQMD data. This is observed for all values $p$. With increasing the values of $\mathrm{p}$ and when is increased the values $\mathrm{q}$ imbalance between the experimental data and UrQMD data increasing. Especially it is typical for $q=4$. Such

Table 1. The correspondence between a sequence number and value of $\operatorname{Ln}(M)$.

\begin{tabular}{|c|c|c|c|}
\hline Sequence number & Value of $\operatorname{Ln}(M)$ & Sequence number & Value of $\operatorname{Ln}(M)$ \\
\hline 1 & 0.693 & 15 & 2.773 \\
\hline 2 & 1.099 & 16 & 2.833 \\
\hline 3 & 1.386 & 17 & 2.890 \\
\hline 4 & 1.609 & 18 & 2.944 \\
\hline 5 & 1.792 & 19 & 2.996 \\
\hline 6 & 1.946 & 20 & 3.045 \\
\hline 7 & 2.079 & 21 & 3.091 \\
\hline 8 & 2.197 & 22 & 3.135 \\
\hline 9 & 2.303 & 23 & 3.178 \\
\hline 10 & 2.398 & 24 & 3.219 \\
\hline 11 & 2.485 & 25 & 3.258 \\
\hline 12 & 2.565 & 26 & 3.296 \\
\hline 13 & 2.639 & 27 & 3.332 \\
\hline 14 & 2.708 & & \\
\hline
\end{tabular}




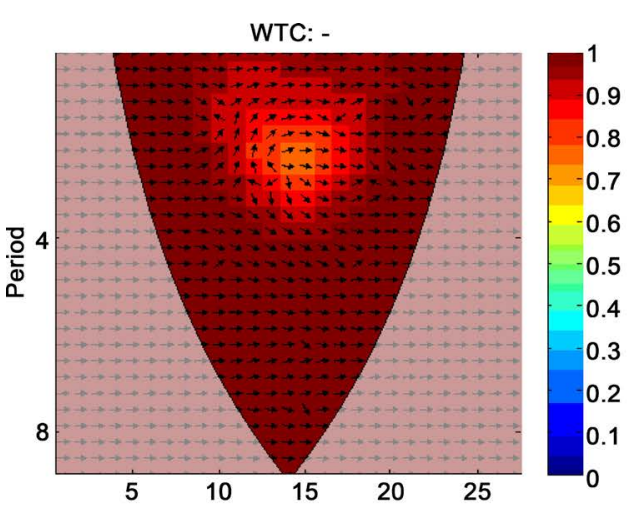

(a)

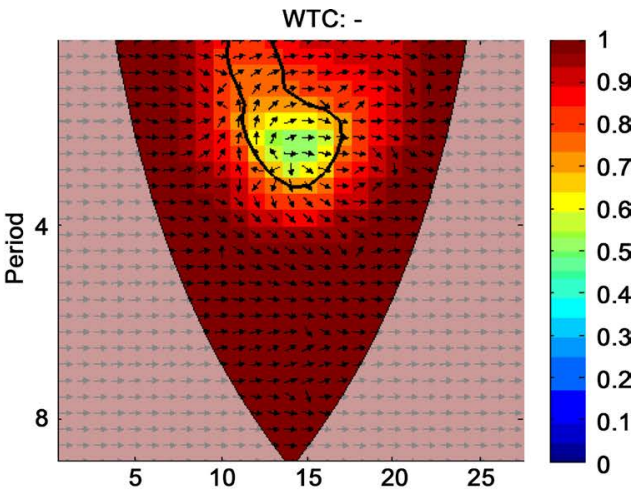

(b)

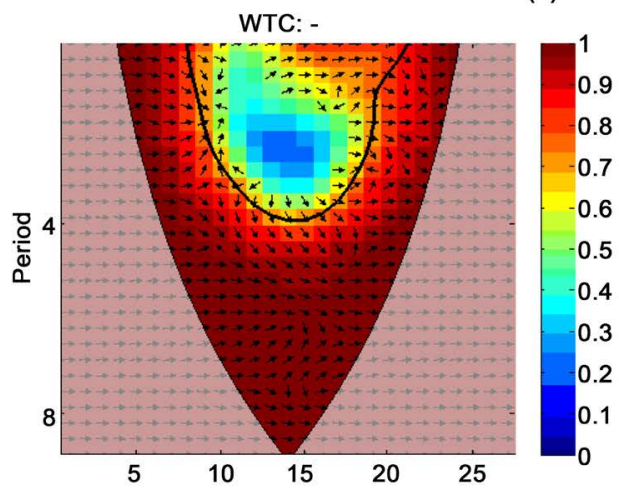

(c)

Figure 2. Wavelet coherence between the experimental data and UrQMD data for $p=0.5$. (a) $q=2$; (b) $q=3$; (c) $q=4$.

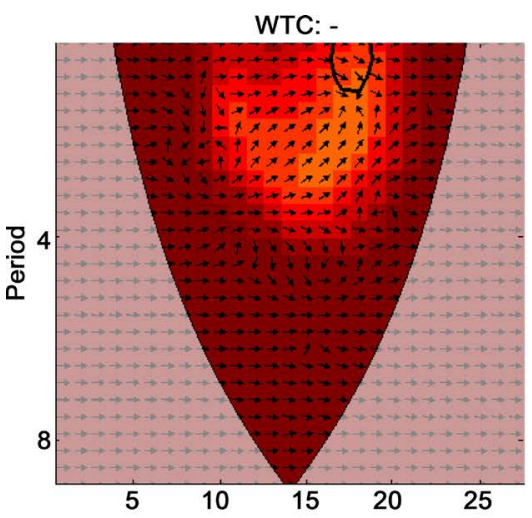

(a)
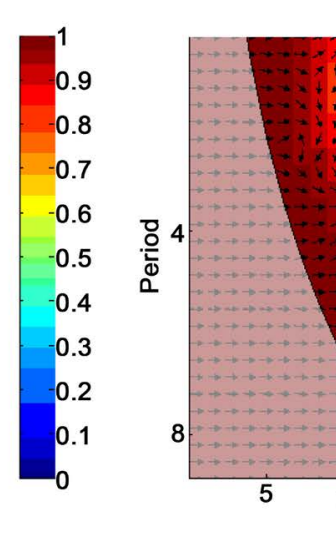

WTC: -

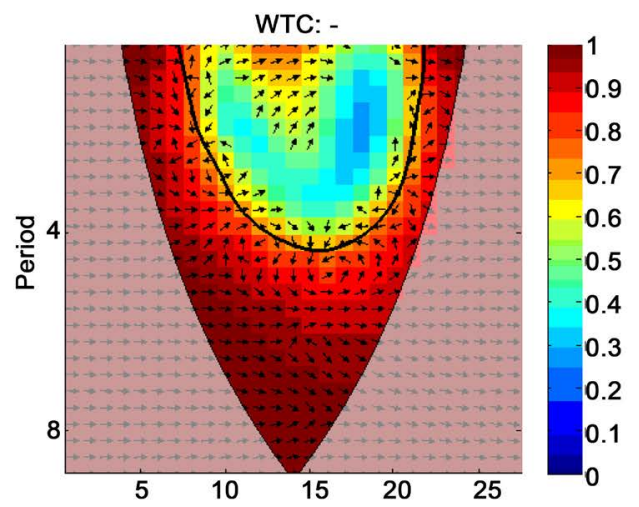

(c)

Figure 3. Wavelet coherence between the experimental data and UrQMD data for $p=0.9$. (a) $q=2$; (b) $q=3$; (c) $q=4$. 


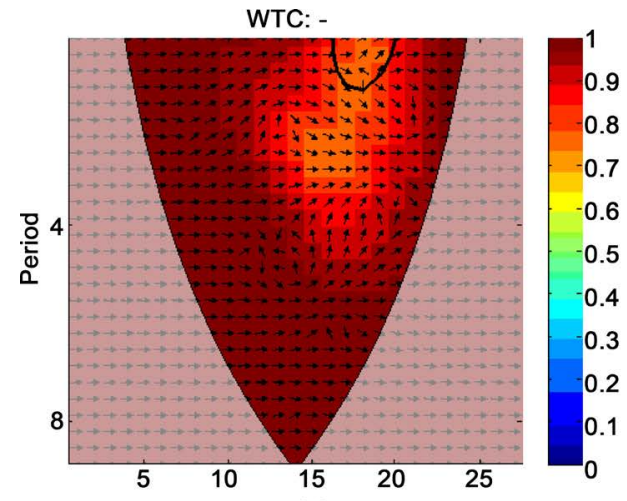

(a)

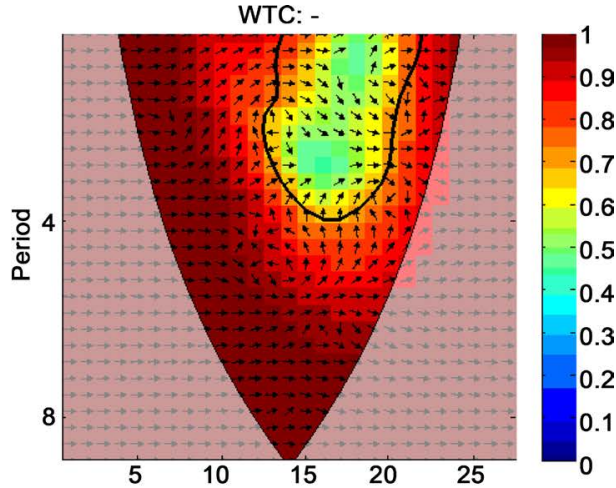

(b)

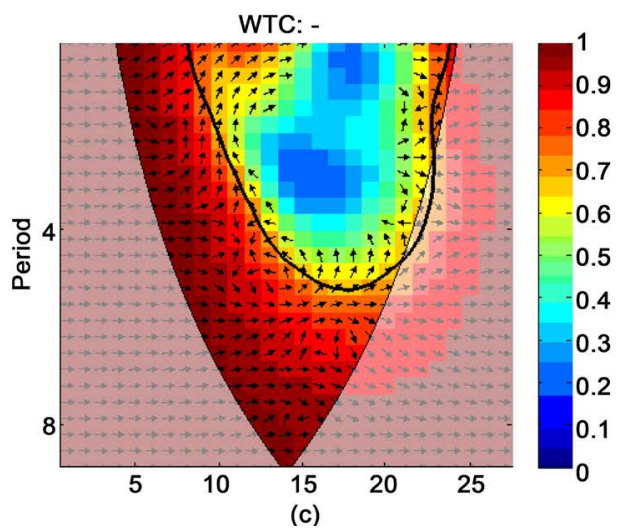

Figure 4. Wavelet coherence between the experimental data and UrQMD data for $p=1.2$. (a) $q=2$; (b) $q=3$; (c) $q=4$.

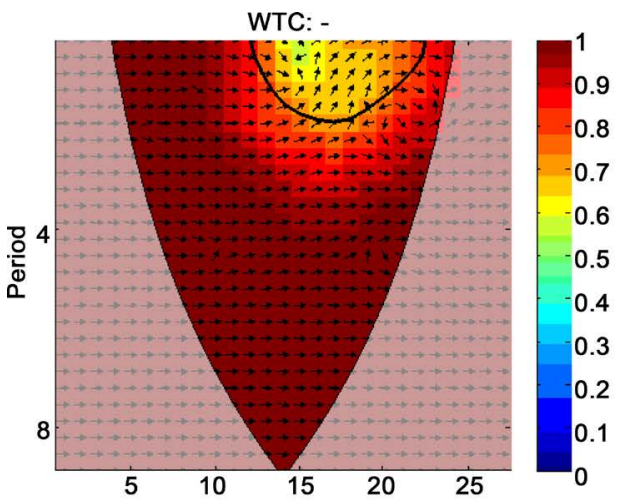

(a)

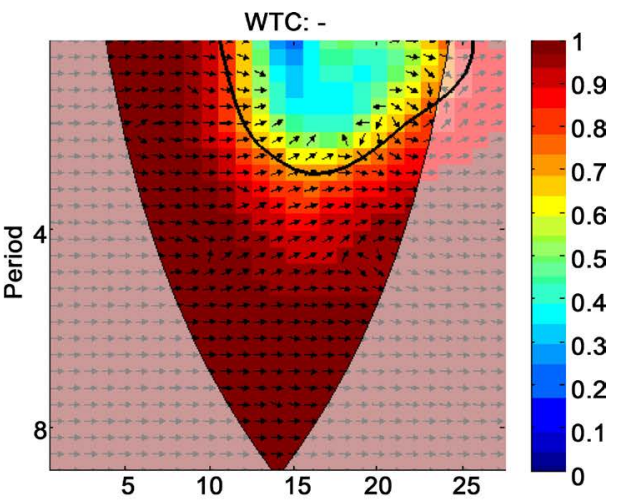

(b)

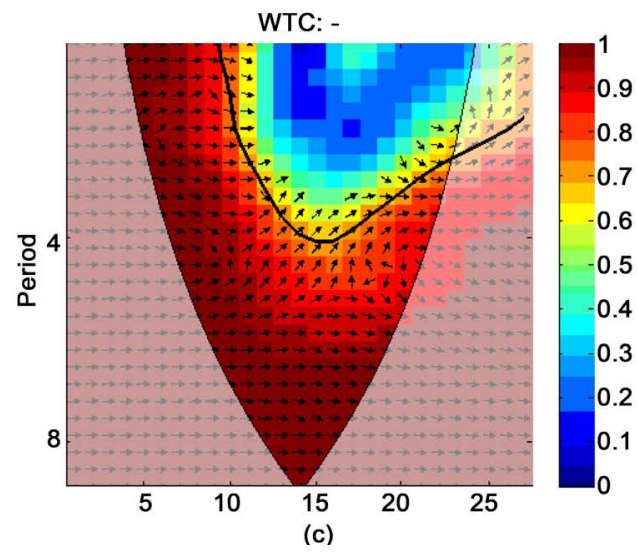

Figure 5. Wavelet coherence between the experimental data and UrQMD data for $p=1.4$. (a) $q=2$; (b) $q=3$; (c) $q=4$. 


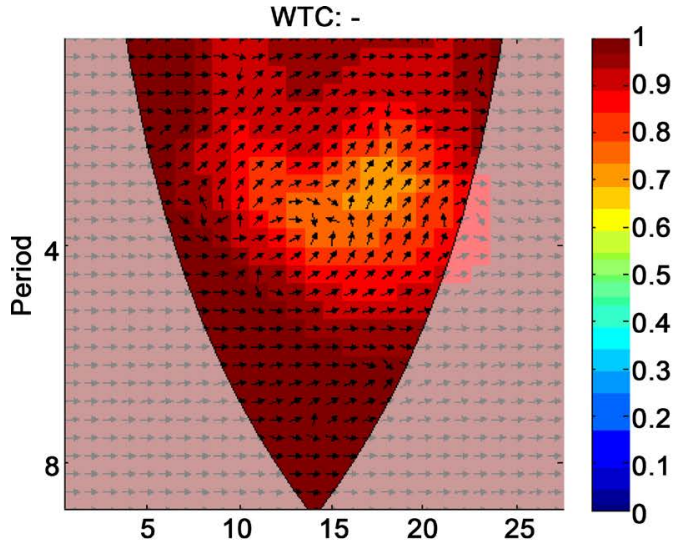

(a)

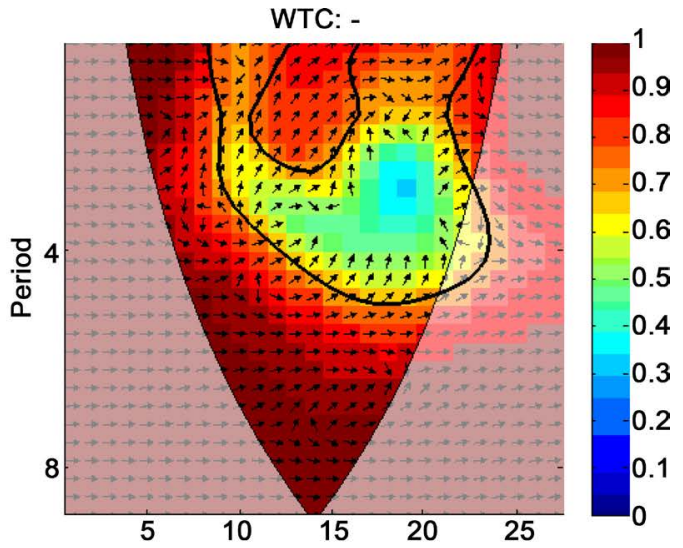

(b)

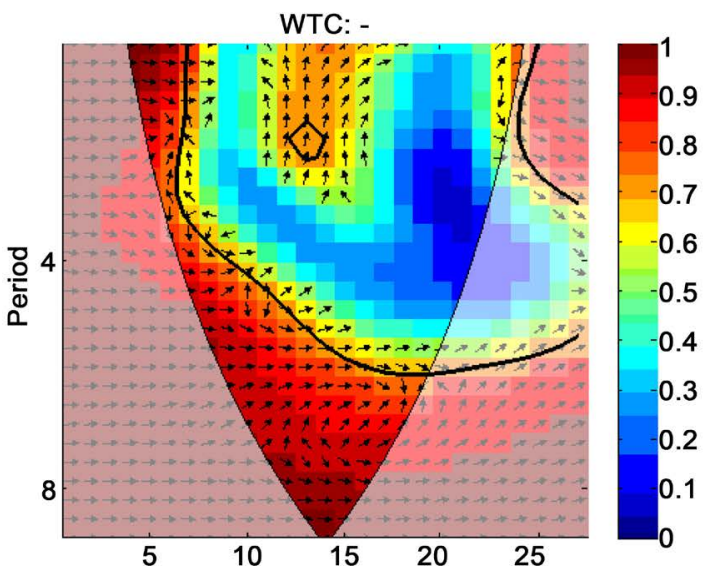

(c)

Figure 6. Wavelet coherence between the experimental data and UrQMD data for $p=1.6$. (a) $q=2$; (b) $q=3$; (c) $q=4$.

an increase is observed for the mean values $\operatorname{Ln}(M)$. At the same time imbalance between the experimental data and UrQMD data in the frequency domain is shifted from the lowest frequency to the average frequency [27]-[32]. Therefore need to have more experimental data to confirm their values. Results which we have obtained are needed to confirm the authenticity of the experiment data and confirm what these data meet certain models.

\section{Conclusions}

The results of the study can determine the appropriate application of wavelet analysis methodology as disclosure of wavelet coherency between the studied data series as a tool for the study collisions of relativistic heavy ion. Some comparative results between the experimental data and UrQMD data have been obtained from the analysis of event-by-event fluctuations of produced charged particles in heavy ion collisions at $14.6 \mathrm{~A} \mathrm{GeV}$. We showed overall consistency between the experimental data and UrQMD data. But we also note that coherence decreases with increasing values of $\mathrm{q}$. This result was obtained for the case $\eta$ -space in 1- $D$ phase space of variable when collisions of relativistic heavy ion. This should be considered when choosing a model for the study and to evaluate the reliability of experimental data. 


\section{Acknowledgements}

The authors would like to acknowledge the keen support for this work of the Department of Physics, Faculty of Science, University of Tabuk, Saudi Arabia and also the Department of Informatics, Kharkiv National University of Radio-Electronics, Kharkiv, Ukraine [22]-[32].

\section{References}

[1] Ahmad, S. and Ahmad, M.A. (2006) A Comparative Study of Multifractal Moments in Relativistic Heavy-Ion Collisions. Journal of Physics G: Nuclear and Particle Physics, 32, 1279-1293. https://doi.org/10.1088/0954-3899/32/9/006

[2] El-Harby, A.A. and Behery, G.M. (2008) Automatic Neural Network System for Vorticity of Square Cylinders with Different Corner Radii. Journal of Applied Mathematics \& Informatics, 26, 911-923.

[3] El-Bakry, M.Y. and El-Metwally, K.A. (2003) Neural Network Model for Proton-Proton Collision at High Energy. Chaos, Solitons \& Fractals, 16, 279-285.

[4] Abou-Moussa, Z. (2002) Compound Multiplicity in the Collisions of $4.1 \mathrm{~A} \mathrm{GeV/c}$ ${ }^{22} \mathrm{Ne}$ Nuclei with Nuclear Emulsion. Canadian Journal of Physics, 80, 109-117. https://doi.org/10.1139/p01-143

[5] Ahmad, M.A., Rasool, M.H., Ahmad, S., Ahmad, N.A. and Madani, J.H. (2013) Chaotic Behaviour of Multiparticle Production in Relativistic Heavy ion Collisions. International Journal of Enhanced Research in Science Technology \& Engineering, 2, 77-89.

[6] Bass, S.A., et al. (1998) Microscopic Models for Ultrarelativistic Heavy Ion Collisions. Progress in Particle and Nuclear Physics, 41, 255-369.

[7] Bleicher, M., et al. (1999) Relativistic Hadron-Hadron Collisions in the Ultra-Relativistic Quantum Molecular Dynamics Model. Journal of Physics G: Nuclear and Particle Physics, 25, 1859-1924. https://doi.org/10.1088/0954-3899/25/9/308

[8] Lyashenko, V., Kobylin, O. and Ahmad, M.A. (2014) General Methodology for Implementation of Image Normalization Procedure Using Its Wavelet Transform. International Journal of Science and Research, 3, 2870-2877.

[9] Lyashenko, V.V., Ahmad, M.A. and Kobylin, O. (2016) Wavelet Analysis as a Learning Tool a Polymer Composites. American Journal of Engineering Research, 6, 1-6.

[10] Lyashenko, V., Deineko, Z. and Ahmad, A. (2015) Properties of Wavelet Coefficients of Self-Similar Time Series. International Journal of Scientific and Engineering Research, 6, 1492-1499.

[11] Lyashenko, V.V., Matarneh, R. and Deineko, Z.V. (2016) Using the Properties of Wavelet Coefficients of Time Series for Image Analysis and Processing. Journal of Computer Sciences and Applications, 4, 27-34.

[12] Rasool, M.H., Ahmad, S. and Ahmad, M.A. (2015) Signal of Unusual Large Fluctuations in ${ }^{32} \mathrm{~S}$-Em Interactions at SPS Energies. Journal of the Korean Physical Society, 67, 448-457. https://doi.org/10.3938/jkps.67.448

[13] Bialas, M. and Gradzicki, M. (1990) A New Variable to Study Intermittency. Physics Letters B, 252, 483-486.

[14] Cao, Z. and Hwa, R.C. (2000) Erraticity Analysis of Multiparticle Production. Physical Review D, 61, Article ID: 074011. https://doi.org/10.1103/PhysRevD.61.074011

[15] Bialas, A. and Czyz, W. (2000) Event by Event Analysis and Entropy of Multipar- 
ticle Systems. Physical Review D, 61, Article ID: 074021. https://doi.org/10.1103/PhysRevD.61.074021

[16] Ghosh, D., Deb, A., Mondal, M. and Ghosh, J. (2003) Erraticity Analysis of Multipion Data in ${ }^{32} \mathrm{~S}-\mathrm{AgBr}$ Interactions at $200 \mathrm{~A} \mathrm{GeV}$. Physical Review $C$, 68, Article ID: 024908. https://doi.org/10.1103/PhysRevC.68.024908

[17] Kingsbury, N. (1999) Image Processing with Complex Wavelets. Philosophical Transactions of the Royal Society of London A: Mathematical, Physical and Engineering Sciences, 357, 2543-2560. https://doi.org/10.1098/rsta.1999.0447

[18] Heil, C.E. and Walnut, D.F. (1989) Continuous and Discrete Wavelet Transforms. SIAM Review, 31, 628-666. https://doi.org/10.1137/1031129

[19] Delbeke, L. and Abry, P. (2000) Stochastic Integral Representation and Properties of the Wavelet Coefficients of Linear Fractional Stable Motion. Stochastic Processes and Their Applications, 86, 177-182.

[20] Torrence, C. and Webster, P.J. (1999) Interdecadal Changes in the ENSO-Monsoon System. Journal of Climate, 12, 2679-2690. https://doi.org/10.1175/1520-0442(1999)012<2679:ICITEM>2.0.CO;2

[21] Grinsted, A., Moore, J.C. and Jevrejeva, S. (2004) Application of the Cross Wavelet Transform and Wavelet Coherence to Geophysical Time Series. Nonlinear Processes in Geophysics, 11, 561-566. https://doi.org/10.5194/npg-11-561-2004

[22] Ahmad S., Ahmad, M.A., Irfan, M. and Zafar, M. (2006) Study of Non-Statistical Fluctuations in Relativistic Nuclear Collisions. Journal of the Physical Society of Japan, 75, Article ID: 064604. https://doi.org/10.1143/JPSJ.75.064604

[23] Ahmad, S. and Ahmad, M.A. (2006) Some Observations Related to Intermittency and Multifractality in ${ }^{28} \mathrm{Si}$ and ${ }^{12} \mathrm{C}-\mathrm{Nucleus}$ Collisiond at $4.5 \mathrm{~A} \mathrm{GeV}$. Nuclear Physics $A, 780,206-221$.

[24] Ahmad, M.A. (2010) A Study of Intermittency and Multifractality in ${ }^{28}$ Si-Emulsion Collisions at 14.6A GeV. PhD Thesis, Aligarh Muslim University, Aligarh, India.

[25] Rasool, M.H., Ahmad, M.A., Bhat, M.A. and Ahmad, S. (2015) Study of Compound Particle Production in ${ }^{28} \mathrm{Si}$ and ${ }^{32}$ Semulsion Collisions at 14.6 and $200 \mathrm{AGeV}$. World Journal of Nuclear Science and Technology, 5, 208-220. https://doi.org/10.4236/wjnst.2015.53021

[26] Tariq, M., Ahmad, M.A., Ahmad, S. and Zafar, M. (2007) Analysis of High NSMultiplicity Events Produced in Relativistic Heavy Ion Collisions at $4.5 \mathrm{~A} \mathrm{GeV/c}$. Romanian Reports in Physics, 59, 773-790.

[27] Rasool, M.H., Ahmad, M.A., Singh, O. and Ahmad, S. (2015) Some Important Features of Relativistic Charged Particles Produced in ${ }^{32} \mathrm{~S}$-Emulsion Interactions at 200 AGeV/c. Journal of Modern Physics, 6, 1498-1509. https://doi.org/10.4236/jmp.2015.611154

[28] Ahmad, M.A., Rasool, M.H., Ahmad, S., Madani, J.H. and Ayad, R. (2013) Study of Deconfinement Phase Transition in Heavy Ion Collisions at BNL Energies. International Journal of Applied Physics and Mathematics, 3, 289-292. https://doi.org/10.7763/ijapm.2013.v3.223

[29] Ahmad, M.A., Rasool, M.H. and Ahmad, S. (2013) Scaling Nature of Target Fragments in the Interactions of ${ }^{28} \mathrm{Si}$-Emulsion at Energy 14.6A GeV. Ukrainian Journal of Physics, 58, 944-955. https://doi.org/10.15407/ujpe58.10.0944

[30] Ahmad, M.A. and Ahmad, S. (2012) Study of Angular Distribution and KNO Scaling in the Collisions of 28Si with Emulsion Nuclei at $14.6 \mathrm{~A} \mathrm{GeV}$. Ukrainian Journal of Physics, 57, 1205-1213.

[31] Rasool, M.H., Ahmad, M.A. and Ahmad, S. (2015) Multifractal Study and Multi- 
fractal Specific Heat of Singly Charged Particles Produced in ${ }^{32} \mathrm{SEm}$ Interactions at 200 AGeV. Chaos Solitons \& Fractal, 81, 197-202.

[32] Rasool, M.H., Ahmad, M.A., Singh, O. and Ahmad, S. (2015) Multiplicities of Forward -Backward Relativistic Charged Particles Produced in 32S-Emulsion Interactions at $200 \mathrm{~A} \mathrm{GeV/c.} \mathrm{Chinese} \mathrm{Journal} \mathrm{of} \mathrm{Physics-Taipei,} \mathrm{53,} \mathrm{100302-100312.}$

\section{Scientific Research Publishing}

Submit or recommend next manuscript to SCIRP and we will provide best service for you:

Accepting pre-submission inquiries through Email, Facebook, LinkedIn, Twitter, etc. A wide selection of journals (inclusive of 9 subjects, more than 200 journals)

Providing 24-hour high-quality service

User-friendly online submission system

Fair and swift peer-review system

Efficient typesetting and proofreading procedure

Display of the result of downloads and visits, as well as the number of cited articles Maximum dissemination of your research work

Submit your manuscript at: http://papersubmission.scirp.org/

Or contact jhepgc@scirp.org 